\title{
Application of Ion-beam Implanted Expanded Polytetrafluoroethylene to Microvascular Decompression and the Surgical Outcome
}

\author{
Koichi Kato, ${ }^{1}$ Hiroshi UjIIE, ${ }^{1}$ Hiroshi NaKanO, ${ }^{1}$ Shunsuke NOMURA, ${ }^{1}$ \\ Masanori NAKAGAWA, ${ }^{1}$ Takashi HigA, ${ }^{1}$ Shigeru KADOYAMA, ${ }^{1}$ \\ and Akira TERAMOTO ${ }^{1}$
}

${ }^{1}$ Department of Neurosurgery, Tokyo Rosai Hospital, Tokyo, Japan

\begin{abstract}
Accurate and long-term transposition of offending vessels is required in microvascular decompression (MVD) for the treatment of hemifacial spasm (HFS) and trigeminal neuralgia (TN). We created ion-beam implanted of an expanded-polytetrafluoroethylene (i-ePTFE) surface to transpose offending vessels in MVD. In 13 patients with MVD, we concealed and transposed offending vessels with tape-shaped i-ePTFE, and relieved facial and trigeminal nerve compression by attaching the i-ePTFE to the dura with fibrin glue. After surgery, none of the patients reported further symptoms or experienced recurrence of symptoms up to 12 months post-surgery. Favorable surgical outcomes are obtainable, since i-ePTFE has high tissue affinity and is easy to manipulate, even under a narrow and deep operative field. Our results suggested that i-ePTFE is very useful for transposition in MVD.
\end{abstract}

Key words: expanded-polytetrafluoroethylene, facial spasm, ion beam, microvascular decompression, trigeminal neuralgia

\section{Introduction}

The use of microvascular decompression (MVD) for the treatment of hemifacial spasm (HFS), trigeminal neuralgia (TN), and glossopharyngeal neuralgia was reported and established by Janneta et al. in $1970 s^{1-7}$. MVD is one of the most cost-effective treatment methods, since patients can withdraw from botox and pharmacological treatments after the surgery. Although there should not be large differences in surgical posture, craniotomy position, and intradural manipulations between facilities, it is important to transpose the offending vessel accurately. Interposition, in which prosthesis is placed between a vessel and a nerve for decompression, should be avoided as much as possible owing to the risk of adhesion, cicatrization, granulomatous changes, and subsequent recurrence of nerve

Received January 13, 2017; Accepted May 16, 2017

Copyright@ 2017 by The Japan Neurosurgical Society This work is licensed under a Creative Commons AttributionNonCommercial-NoDerivatives International License. compression $^{7-9)}$. For the transposition of vessels, cord-shaped teflon felt (Sly Inc., Strongsville, $\mathrm{OH}, \mathrm{USA}$ ) and tape-shaped gore-tex (W.L. Gore \& Associates, Inc., Newark, DE, USA) are used to lift the vessel and are adhered to the dura with fibrin glue. However, expanded-polytetrafluoroethylene (ePTFE), which consists of both teflon felt and gore-tex, is hydrophobic and only weakly adheres to the dura with fibrin glue. Thus, there is a risk of detachment from the dura after surgery when the bond is exposed to cerebrospinal fluid (CSF). CSF leakage is also a common complication after MVD when ePTFE is used for dural sutures. To address these limitations, we developed ion-beam implanted ePTFE (i-ePTFE) with tissue bondability. We improved the surface of the ePTFE by focusing a radiating ion-beam. We applied i-ePTFE to cerebral aneurysm wrapping, artificial dura mater, and vascular prosthesis following approval of the ethical committee ${ }^{10,11)}$. In this study, we investigated the manipulability, post-operative complications, symptom recurrence, and effectiveness of i-ePTFE in patients undergoing MVD. 


\section{Surgical Methods}

Our hospital adopted standardized surgical procedures for MVD. We performed a 3-pins fixation with the patient positioned such that the impaired side was elevated and the head was rotated to the non-affected side and mildly flexed so that the mastoid process was in a higher position. The skin incision was a mild S-shape extending from the height of postauricular region to the first cervical vertebra, where the incision height in TN was 5-10 millimeters higher than that in HFS. For craniotomy, one burr hole was fixed and the dura mater was separated from the bone with a dissector, resulting in a bone incision of $2.5-3 \mathrm{~cm}$. Additional bone was removed until the medial edges of the transverse sinus and sigmoid sinus were exposed in $\mathrm{TN}$ and the caudal sigmoid sinus was exposed in HFS. After the dural incision, CSF was suctioned to slacken the cerebellum, and we approached the root exit (entry) zone of the nerve from the higher lateral side of the cerebellum and the lower cranial nerve side in TN and HFS, respectively. In $\mathrm{TN}$, the offending vessel was transposed after assuring sufficient mobility by adequately resecting the arachnoid membrane around the nerve and petrosal vein. After catching the offending vessel with $100 \mu \mathrm{m}$-thick i-ePTFE, fixation with fibrin glue was performed to the tentorium cerebelli or pyramidal bone in TN and HFS, respectively. Auditory brainstem response (ABR) and abnormal muscle response (AMR) were continuously monitored. When the $\mathrm{V}$ wave of $\mathrm{ABR}$ was prolonged for $1 \mathrm{~ms}$, traction of the cerebellum was stopped and the procedure was not resumed until after we confirmed that the latency recovered to $0.5-0.6 \mathrm{~ms}$. After nerve decompression, the dura was densely sutured and the bone fragment was fixed to the surrounding bones using titanium plates.

\section{Subjects and Methods}

The study participants included 13 MVD patients who underwent transposition of offending vessels with i-ePTFE between January 2011 and January 2016. Ten female and three male patients participated in this study (average age: 57.1 years (range: 30-79 years); 9 TN, 4 HFS).

We investigated the offending vessels, post-surgical complications including temporal complications, and recurrence to evaluate the effectiveness of i-ePTFE in MVD.

\section{Results}

No prostheses were used between the nerves and vessels and transposition of the offending vessels was possible in all of the patients. The offending vessels in TN included the superior cerebellar artery (SCA) in six cases, the petrosal vein in two cases, and the vertebral artery (VA) in one case, including duplicates (Table 1). The offending vessels in HFS were the VA

Table 1 Summary of the patients treated by MVD with ion-beam implanted ePTFE

\begin{tabular}{ccclll}
\hline Case & Age & Sex & Diagnosis & Offending vessel & Complications \\
\hline 1 & 70 & F & rt facial spasm & rt AICA & None \\
2 & 79 & F & rt trigeminal neuralgia & rt SCA & None \\
3 & 77 & F & rt trigeminal neuralgia & rt AICA & None \\
4 & 77 & F & rt trigeminal neuralgia & rt VA & None \\
5 & 56 & F & rt trigeminal neuralgia & rt SCA, rt petrous vein & None \\
6 & 53 & F & rt trigeminal neuralgia & rt petrosal vein & None \\
7 & 42 & M & rt trigeminal neuralgia & rt SCA & None \\
8 & 67 & F & lt trigeminal neuralgia & lt SCA & None \\
9 & 70 & F & lt trigeminal neuralgia & lt SCA & None \\
10 & 30 & M & rt facial spasm & rt PICA & None \\
11 & 62 & F & lt trigeminal neuralgia & lt SCA & Transient facial \\
12 & 65 & M & lt facial spasm & lt VA & numbness \\
13 & 54 & F & lt facial spasm & lt VA, lt AICA & Transient hoarseness \\
\hline
\end{tabular}

AICA: anterior inferior cerebellar artery, PICA: posterior inferior cerebellar artery, SCA: superior cerebellar artery, VA, vertebral artery. 
in one case, the anterior inferior cerebellar artery (AICA) in one case, VA and AICA in one case and the posterior inferior cerebellar artery (PICA) in one case.

Although temporal numbness in the facial region and hoarseness occurred after surgery in one patient with TN and another patient with HFS, respectively, no permanent complications occurred, and all patients were able to withdraw from pharmacological and botox treatments.

There was no recurrence of symptoms at 12 months to 6 years post-surgery.

\section{Representative Case}

Sixty-five-year-old male Although he had undergone 3 years of effective botox treatment for left HFS, he elected to have surgery rather than continued botox treatments in the future. An magnetic resonance imaging/angiography (MRI/A) of the brain showed a bilateral meandering VA compressing the root of the left facial nerve (Figs. 1A and 1B).

In the surgical findings, the bilateral meandering VA was located in parallel, and the left VA beside the VA union adjoined the exit zone of the left facial nerve from rostral side. Arteriosclerosis in the left VA
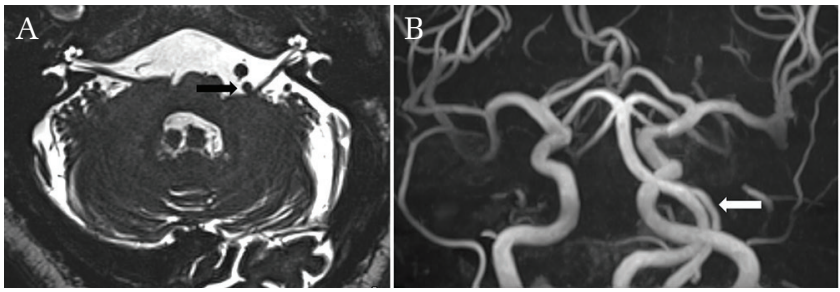

Fig. 1 Brain MRI (A) and MRI (B) shows bilateral meandering vertebral arteries compressing the root of the left facial nerve. was severe and it promptly returned to its original position even when moved by tweezers. First, the left VA was wrapped in the i-ePTFE (Fig. 2A), which was trimmed to a width of $4 \mathrm{~mm}$ and a length of $25 \mathrm{~mm}$, and adhered to the dura of the pyramidal bone with fibrin glue (Fig. 2B). It was presumed that transposition of the right VA, which compressed the left VA, was required. The right and left VAs were wrapped in the i-ePTFE, which was trimmed to a width of $3 \mathrm{~mm}$ and a length of $30 \mathrm{~mm}$, and transposed to the dura with fibrin glue to reinforce the transposition in the range where penetrating branches to brainstem did not diverge (Fig. 2C). After maintaining dry conditions for a few minutes and confirming that the vessel was stabilized, artificial CSF was added to the surgery field to confirm that the i-ePTFE did not detach in wet conditions and that the VA remained adhered to the pyramidal bone. Then, the operative field was closed.

Although left HFS disappeared, temporal hoarseness occurred. The hoarseness lasted 2 weeks; however, the patient gradually recovered. A postoperative MRI of the brain showed the successful transposition of the bilateral VAs from the brainstem to the dura (Fig. 3).

\section{Discussion}

It is possible to trim i-ePTFE into a desired size, making the preparation during the MVD surgery. Additionally, it is easy to wrap i-ePTFE around deep vessels without damaging the surrounding vessels even with a narrow surgery field. Even when strong traction is required owing to arteriosclerosis and meandering of the VA, strong adherence to the dura can still be obtained without suture ${ }^{12)}$.
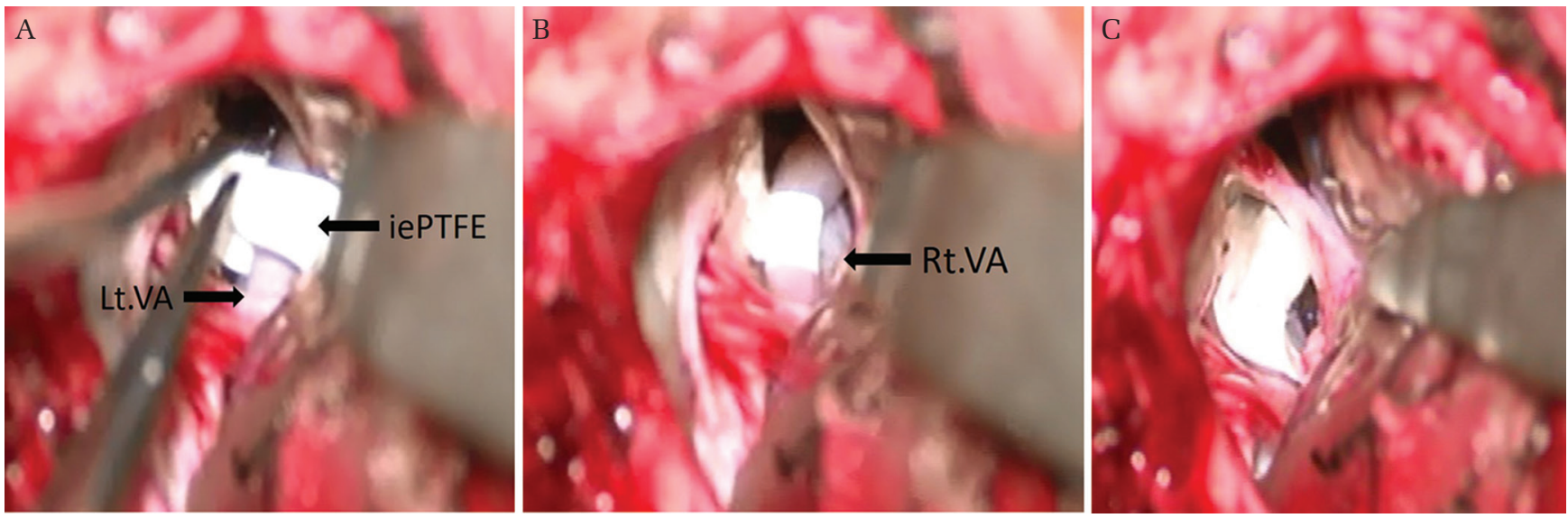

Fig. 2 The left VA is wrapped with ion-beam irradiated expanded polytetrafluoroethylene (i-ePTFE) (A) and is adhered to the dura of the pyramidal bone with fibrin glue (B). The right and left VAs is wrapped with i-ePTFE and is attached to the dura with fibrin glue (C). 


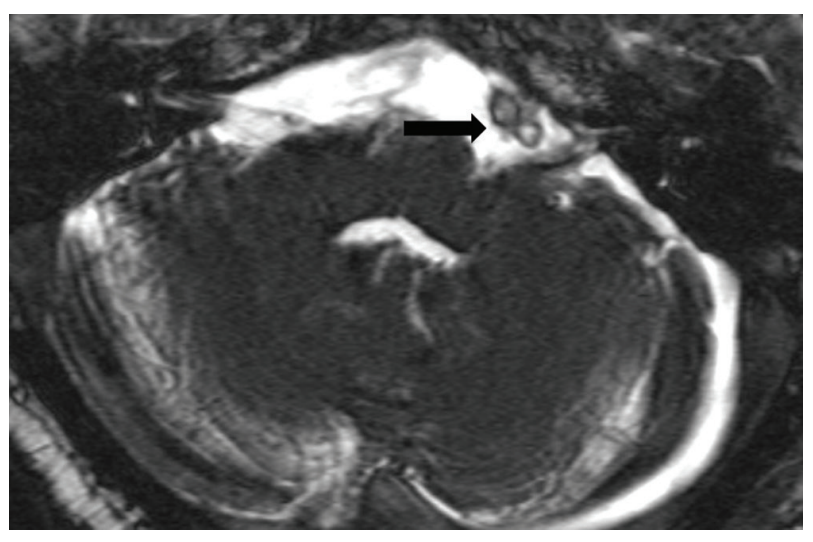

Fig. 3 Postoperative brain MRI shows the bilateral VAs transposed from the brainstem.

Among all the participants in this study, only 2 patients experienced post-operative complications. The facial numbness that occurred after the TN surgery was likely due to traction of the proximal facial nerve, while it was far from the trigeminal nerve. Furthermore, hoarseness, which occurred in the representative patient case shown above after the HFS surgery, was presumably due to the lack of careful manipulation of the lower cranial nerves. Fortunately, post-surgical complications in both of the patients were temporal and eventually resolved. When surgical outcomes are evaluated on the basis of the MVD post-operative outcome assessment ${ }^{13)}$, good to excellent outcomes were obtained in all patients.

The complete cure rate in MVD is $70-80 \%$ and $84-92 \%$ for TN and HFS, respectively ${ }^{7}$. The offending vessels are transposed to the tentorium cerebelli in $\mathrm{TN}$, while the vessels are sometimes pulled toward the lower cranial nerves in HFS. Hence, it is also important not to damage the IX and X cranial nerves during surgical manipulations. The optimal direction to provide stability while avoiding excessive flexion depends on the location of the offending and surrounding vessels. Transposition becomes difficult when 1) the compression is due to dolichoectasia in the VA, 2) the compression is due to the AICA located between the facial and auditory nerves, 3) and when many penetrating branches to brainstem are present.

We formerly adhered cord-shaped teflon felt to the dura using fibrin glue and achieved low rates of recurrence, which demonstrated that sufficient transposition was obtained for offending vessels in most patients. However, strong traction is required for the VA and meandering and thick vessels; even after wrapping the teflon felt onto the offending vessels twice or three times and adhering it to the dura using fibrin glue, we were still concerned about post-surgery detachment. The adhesion of regular ePTFE and fibrin glue decreases in wet environments. With i-ePTFE, the surface of the ePTFE is transformed using an ion-beam to avoid the smooth surface, which increases the adhesion of the fibrin glue and enables accurate transposition of the offending vessels. The cord-shaped teflon felt carries the risk of 1) being caught by fine penetrating branches owing to fray during wrapping onto vessels and 2) reduction of adhesion due to exposure to CSF before being adhered to the dura. When symptoms reappear after transposition to the dura using teflon felt or gore-tex with fibrin glue, it is likely owing to detachment of the compressive vessels from the dura that can occur when the fibrin glue decomposes and disappears in CSF. Patients who underwent MVD with i-ePTFE as traction tape did not experience recurrence of symptoms and had favorable outcomes, suggesting that the transposition of the offending vessels remained long-term. i-ePTFE can be manipulated easily and can be used to wrap aneurysms and repair damage in vessels in combination with fibrin glue ${ }^{10,11)}$. We believe that i-ePTFE is very useful, since it can also reinforce areas where suture during dura damage is difficult.

Since stable and long-term transposition of offending vessels can prevent recurrence, various attempts have been reported after the initial report of the MVD with ePTFE by Fukushima ${ }^{5}$. Tanaka et al. introduced a surgical method where they wrapped belt-shaped ePTFE onto offending vessels and sutured it to the dura ${ }^{14)}$. Ogawa et al. also introduced a method where they lifted the VA with ePTFE and sutured it to the dura with 6-0 nylon ${ }^{15}$. Although it is unlikely that ePTFE splits apart owing to the penetrating needle into the ePTFE, it is possible that it remains unstable when the ability of the vessel to return to its original position is strong. When ePTFE can be adhered using fibrin glue without a suture, the operative procedures are even simpler. Furthermore, more stable transposition of thick vessels with more restoring force can be obtained by adding sutures with string.

ePTFE is produced by PTFE, and its molecular structure consists of fluorine atoms evenly covering and protecting the carbon atom chains; thus, it is very chemically stable inside biological organisms, unlike polyethylene, polypropylene, and polystyrene. ePTFE also has very low reactivity to tissues $^{10-12)}$. Therefore, ePTFE is applied to a wide range of biomaterials, such as vascular prostheses, artificial cardiac membranes, artificial peritoneum, and screen membranes for guided tissue regeneration $^{16)}$. However, while the low tissue reactivity is advantageous, it can also be disadvantageous as 


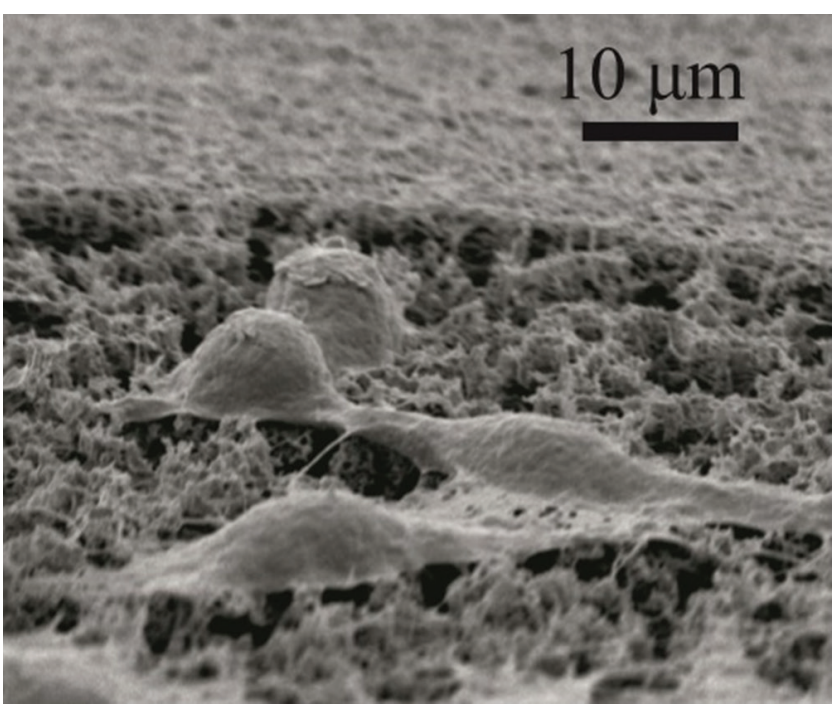

Fig. 4 Electron microscopy showing the fibroblast cell attachment to ion-beam $\left(\mathrm{Ar}^{+}\right.$ion) implanted ePTFE.

follows: 1) it can cause CSF leakage (which occurs in the gap between ePTFE and surrounding tissues and the punctuation hole made during suture), and 2) it does not coalesce with biological tissues.

The ion-beam implanted technique ionizes targeted substances in a high vacuum environment (10-40 $\mathrm{Pa}$ ). Then, the substances are embedded to a solid substrate $^{12)}$. Since the accelerated ion-beam is massseparated, the ion purity becomes extremely high. Furthermore, the controllability of the ion-beam irradiation technique, such as the acceleration energy and irradiance level, is also high, which enables processing with high reproducibility and uniformity. It is possible to only reform the surface (surface reforming) by taking advantage of the dynamic characteristics of the material to improve biocompatibility such as cellular adhesiveness. The i-ePTFE in our study used ionized argon, which is a safe substance as an inactive gas that does not remain in the material. i-ePTFE is adhered to biological tissues using fibrin glue because of the surface reforming and also developing fibroblast cells between them (Fig. 4). Furthermore, the thickness of commercialized gore-tex is $300 \mu \mathrm{M}$, while that of our i-ePTFE is $100 \mu \mathrm{M}$, which allowed us to wrap vessels flexibly without damaging the surrounding penetrating branches or being repelled. Our results demonstrated that MVD with i-ePTFE is a safe and accurate surgical procedure.

\section{Conclusion}

i-ePTFE adds compatibility such as cellular adhesiveness by reforming only surface by taking advantage of dynamic characteristics of the material. The adhesiveness of fibrin glue is improved with i-ePTFE, and there is less risk of detachment once it is adhered to the dura, even in wet environments. It can be quickly adjusted to an optimal size during surgery and can be easily manipulated to wrap around vessels. Our results showed that i-ePTFE might be a suitable material for the transposition of offending vessels in MVD, considering that there was no recurrence in our patients and the long-term clinical course was favorable.

\section{Conflicts of Interest Disclosure}

The authors report no conflict of interest.

\section{References}

1) Jannetta PJ: Microsurgical approach to trigeminal nerve for tic doulourex. Prog Neurol Surg 7: 180-200, 1976

2) Jannetta PJ, Abbasy M, Maroon JC, Ramos FM, Albin MS: Etiology and definitive microsurgical treatment of hemifacial spasm. Operative techniques and results in 47 patients. J Neurosurg 47: 321-328, 1977

3) Jannetta PJ: Microsurgery of cranial nerve crosscompression. Clin Neurosurg 26: 607-615, 1979

4) Kondo A, Ishikawa J, Yamasaki T, Konishi T: Microvascular decompression of cranial nerves, particularly 7th cranial nerve. Neurol Med Chir (Tokyo) 20: 739-751, 1980

5) Fukushima T: [Results of posterior fossa microvascular decompression in the treatment of hemifacial spasm]. Facial N Res Jpn 4: 9-14, 1984 (Japanese)

6) Matsushima T, Fukui M, Suzuki S, Rhoton AL: The microsurgical anatomy of the infratentorial lateral supracerebellar approach to the trigeminal nerve for tic douloureux. Neurosurgery 24: 890-895, 1989

7) Matsushima T, Mineta T: [Microvascular compression syndrome: review and update of microvascular decompression surgery]. No Shinkei Geka 36: 303-313, 2008 (Japanese)

8) Chen J, Lee S, Lui T, Yeh Y, Chen T, Tzaan W: Teflon granuloma after microvascular decompression for trigeminal neuralgia. Surg Neurol 53: 281-287, 2000

9) Smucker P, Bonnin JM, Pritz MB: Teflon granuloma with midbrain cysts after microvascular decompression of the trigeminal nerve. Acta Neurochir (Wien) 149: 537-539, 2007

10) Takahashi N, Ujiie H, Suzuki Y, Iwaki M, Hori T: [Biocompatibility of ePTFE modified by ion beam irradiation]. No Shinkei Geka 32: 339-344, 2004 (Japanese)

11) Ujiie H, Higa $T$, Katoh $K$, et al.: [Wrap-clipping with ion beam irradiated ePTFE]. Surg Cereb Stroke (Japanese) 35: 24-29, 2007 
12) Suzuki Y, Iwaki M, Tani S, Oohashi G, Kamio M: Ion implantation into ePTFE for application of an artificial dural substitute. Nucl Instr Meth B206: 538-542, 2003

13) Kondo A: [A proposal for standardizing the results of microvascular decompression surgery]. Jpn J Neurosurg 19: 691-695, 2010 (Japanese)

14) Tanaka Y, Uchida M, Onodera H, Hiramoto J, Yoshida Y: Simple transposition technique for microvascular decompression using an expanded polytetrafluoroethylene "Belt": Technical note. Neurol Med Chir (Tokyo) 54: 483-485, 2014

15) Ogawa A, Suzuki M, Shirane R, Yoshimoto T: Repositioning of the tortuous vertebrobasilar artery for trigeminal neuralgia: a technical note. Surg Neurol 38: 232-235, 1992

16) Wilson SE, Jabour A, Stone RT, Stanley TM: Patency of biologic and prosthetic inferior vena cava grafts with distal limb fistula. Arch Surg 113: 1174-1179, 1978

Address reprint requests to: Koichi Kato, MD, Department of Neurosurgery, Tokyo Rosai Hospital, 4-31-21 Omoriminami, Ota-ku, Tokyo 143-0013, Japan. e-mail: kkato@tokyoh.johas.go.jp 